\title{
Metastable biomolecular condensates of interferon-inducible antiviral Mx-family GTPases: A paradigm shift in the last three years
}

\author{
Pravin B Sehgal (0) \\ Departments of Cell Biology and Anatomy, and Medicine, \\ New York Medical College, Valhalla, NY, USA \\ (Email,pravin_sehgal@nymc.edu) \\ MS received 17 December 2020; accepted 5 June 2021
}

\begin{abstract}
Membraneless organelles (MLOs) in the cytoplasm and nucleus in the form of phase-separated biomolecular condensates are increasingly viewed as critical in regulating diverse cellular functions. We summarize a paradigm shift over the last 3 years in the field of interferon (IFN)-inducible antiviral Mx-family GTPases. Expression of the 'myxovirus resistance proteins' MxA in human cells and its ortholog Mx1 in murine cells is increased 50- to 100 -fold by Type I (IFN- $\alpha$ and $-\beta$ ) and III IFNs (IFN- $\lambda$ ). Human MxA forms cytoplasmic structures, while murine Mx1 forms nuclear bodies. Since 2002, it has been widely thought that human (Hu) MxA is associated with the membraneous smooth endoplasmic reticulum (ER). In a paradigm shift, our recent data showed that HuMxA formed membraneless phase-separated biomolecular condensates in the cytoplasm. Some of the HuMxA condensates adhered to intermediate filaments generating a reticular pattern. Murine ( $\mathrm{Mu}) \mathrm{Mx} 1$, which was predominantly nuclear, was also confirmed to be in phase-separated nuclear biomolecular condensates. A subset of Huh7 cells showed association of GFP-MuMx 1 with intermediate filaments in the cytoplasm. While cells with cytoplasmic GFP-HuMxA condensates and cytoplasmic GFP-MuMx1 filaments showed an antiviral phenotype towards vesicular stomatitis virus (VSV), those with only nuclear GFP-MuMx1 bodies did not. The new data bring forward the paradigm that both human MxA and murine Mx1 give rise to phase-separated biomolecular condensates, albeit in different subcellular compartments, and that differences in the subcellular localization of condensates of different Mx proteins determines the spectrum of their antiviral activity.
\end{abstract}

Keywords. Interferons; myxovirus resistance proteins; cytoplasmic human MxA condensates; nuclear and cytoplasmic murine Mx1 condensates; subcellular localization; antiviral activity; tonicity- and crowding-driven metastability

\section{Introduction}

In the process of investigating a phenomenon called 'viral interference' in which one virus interferes with the replication of another related or even unrelated virus, Isaacs and Lindenmann observed in 1957 that chicken embryo tissue in cell culture exposed to live or

This article is dedicated to the memory of the late Dr. Manohar V. Shirodkar, Director, The Haffkine Institute, Mumbai, India, who taught the author to go wherever the data led. ultraviolet-irradiated influenza virus type A (FLUAV, an RNA-containing myxovirus isolated from humans) secreted a protein into the culture medium which could render fresh cultures of chicken tissue resistant to a challenge with live FLUAV (Isaacs and Lindenmann 1957; Isaacs et al. 1957). This protein, which elicited an antiviral effect against FLUAV, was called interferon' (IFN). Subsequently, Lindenmann investigated human-derived FLUAV strain adapted to mice and observed that while the A2G strain of inbred mice, and outbred and field mice were resistant to the production of pulmonary disease when FLUAV was administered 
by inhalation, other inbred mouse strains (e.g. Balb/c) were susceptible (Lindenmann 1962; Lindenmann et al. 1963). It was soon recognized that this resistance to inhaled FLUAV was due to a genetic trait dubbed ' $M x$ ' for 'myxovirus resistance gene.' (Lindenmann et al. 1963; Lindenmann 1964; Haller et al. 2015, 2018).

Contemporaneously with these studies, numerous investigators examined the ability of one virus to inhibit development of disease by other disparate viruses in animal models (Henle 1950; Ho 1962). One such investigation, initiated in the early 1960 s at the Johns Hopkins School of Public Health and continued at the Virus Research Center, Pune (now called the National Institute of Virology) showed that West Nile virus inoculation of chicken could block the development of sarcoma in the wing web following a challenge with the Rous sarcoma virus (Shirodkar 1965). Subsequently it was discovered that a vaccine strain of rabies virus was also able to cause this blockade of the Rous sarcoma in chicken (Desai 1970), and that the plasma/serum of rabies virusinoculated chicken contained a protein which blocked sarcoma development produced by the Rous virus (Desai et al. 1973). Taken together, an interpretation of these observations was the possibility that virusinduced interferon mediated this anti-tumor effect (Shirodkar 1965; Desai 1970; Desai et al. 1973). Parenthetically, the present author received his first detailed education about the interferons through participation with Dr. M. V. N. Shirodkar in some of these studies (Desai et al. 1973).

Evidence for the involvement of interferon (IFN) in vivo in mice resistant to an inhaled challenge of FLUAV was reported in 1979 by Haller and colleagues (as part of the Lindenmann research group) (Haller et al. 1979). An antibody that neutralized mouse IFN administered to $\mathrm{A} 2 \mathrm{G}$ mice rendered such resistant mice susceptible to development of viral pneumonia. Thus, the ' $M x$ ' resistance gene produced an IFN-inducible gene product with antiviral activity against FLUAV and other myxo- and paramyxoviruses. Over the next two decades, in very elegant studies, Haller and colleagues, identified the mouse Mx1 and as well as human MxA proteins as $60-70 \mathrm{kDa}$ dynamin-family large GTPases which had an antiviral effect against a variety of different RNA- and DNA-containing viruses (table 1; Verhelst et al. 2013; Haller et al. 2015 2018). Exposure to cells with appropriate IFN preparations upregulated Mx RNA and protein expression by 50 - to 100 -fold in human and mouse cells (Haller et al. 2015 2018; Yuan and Sehgal 2016). Moreover, a second human Mx gene
(' $M x B$ ') was discovered, as was a second mouse $\mathrm{Mx}$ gene ('Mx2') (Haller et al. 2015, 2018). Phylogenetic analyses showed that $M x A$ and $M x B$ represented distinct genes, but that murine $M x 1$ and $M x 2$ were orthologs of $M x A$ (the rodent ortholog of $M x B$ had been lost) (table 1; Busnadiego et al. 2014; Haller et al. 2015). Human MxA and MxB, and murine Mx1 and Mx2 proteins had distinct subcellular localizations (cytoplasmic vs. nuclear) and distinct antiviral activities towards different viruses (table 1; see Verhelst et al. 2013; Haller et al. 2015, 2018, Steiner and Pavlovic 2020 for detailed reviews).

Briefly, human MxA formed cytoplasmic structures, human MxB was located in structures near the nuclear pores, murine Mx1 formed nuclear structures, while murine Mx2 was 'granular' and in the cytoplasm. Additionally, human MxA was antiviral towards FLUAV and vesicular stomatitis virus (VSV, a rhabdovirus whose entire life cycle is cytoplasmic), while human $\mathrm{MxB}$ was antiviral towards lentiviruses such as HIV but not FLUAV or VSV. In contrast to human MxA, the orthologous nuclear murine Mx1 was antiviral towards FLUAV (which has an obligatory nuclear step in its life cycle) but not against VSV, while, curiously, the cytoplasmic granular murine Mx2 (also an ortholog of human MxA) was antiviral towards VSV (Haller et al. 2015; Sehgal et al. 2020b) (table 1). The GTPase activity was required for these antiviral activities (Dick et al. 2015; Nigg and Pavlovic 2015; Haller et al. 2015, 2018). However, despite extensive studies over the last six decades, the molecular mechanisms by which Mx proteins inhibit replication of different viruses in intact cells are incompletely understood (Haller et al. 2015, 2018; Sehgal et al. 2020b; Steiner and Pavlovic 2020). Surprisingly, even the understanding of the basic cell biology of Mx proteins needed a paradigm shift in the last three years.

\section{A paradigm shift in the Mx field in last three years}

In 2002 the Haller group (Kochs et al. 2002) reported that electron microscopy of cells transfected with human MxA expression vector together with infection by La Crosse virus revealed juxtanuclear membraneless structures which contained the viral nucleocapsid (N) protein and MxA. In this article in 2002, Haller and colleagues stated specifically that these structures did not associate with any intracellular membranes (Kochs et al. 2002). 
Table 1. Condensates of different human and murine Mx proteins

\begin{tabular}{|c|c|c|c|}
\hline Protein & Cellular localization & $\begin{array}{c}\text { Illustrative } \\
\text { Antiviral activity* }\end{array}$ & References \\
\hline Human MxA & $\begin{array}{l}\text { Cytoplasmic condensates with some } \\
\text { tethered to intermediate filaments }\end{array}$ & vs. both FLUAV and VSV & Davis et al. $(2018,2019)$ \\
\hline $\begin{array}{r}\text { Human MxB } \\
\text { (full length) }\end{array}$ & Cytoplasmic face of nuclear pores, & vs. HIV; not vs. FLUAV nor VSV & $\begin{array}{l}\text { King et al. (2004) } \\
\text { Goujon et al. (2013) }\end{array}$ \\
\hline Murine Mx1 & $\begin{array}{l}\text { Cytoplasmic condensates } \\
\text { nuclear condensates } \\
\text { cytoplasmic intermediate filaments and } \\
\text { condensates }\end{array}$ & $\begin{array}{l}\text { vs. FLUAV; not VSV } \\
\text { vs. VSV }\end{array}$ & $\begin{array}{l}\text { our inference } \\
\text { Sehgal et al. }(2020 \mathrm{~b}) \\
\text { Sehgal et al. }(2020 \mathrm{~b})\end{array}$ \\
\hline Murine Mx2 & Cytoplasmic structures & vs. VSV; not FLUAV & Haller et al. (2015) \\
\hline
\end{tabular}

*See reviews Verhelst et al. (2013), Haller et al. (2015, 2018) and Steiner and Pavlovic (2020) for detailed antiviral spectrum reported in the previous literature.

Modified from Sehgal et al. (2020b).

In contrast, also in 2002, McNevin and colleagues (Accola et al. 2002) claimed that MxA expressed in Hela and Hep3B cells was associated with the smooth endoplasmic reticulum (ER). However, in the article itself, these authors admitted that they were unable to associate MxA with any customary markers for the ER or any other membrane-bound organelles, and that the association was 'presumed' to be with the ER because of an impression that MxA colocalized with the 'autocrine motility factor receptor' (AMF), which they claimed had been assigned by them to the ER. Nevertheless, the abstract dropped the 'presumably' and the title of Accola et al. (2002) stated categorically that $\mathrm{MxA}$ was associated with the smooth ER.

In 2006, Haller and colleagues (Stertz et al. 2006) appear to have adjusted their earlier inference from 2002 that MxA structures were membraneless (as in Kochs et al. 2002) and now claimed that MxA associated with a 'distinct subcompartment of the smooth endoplasmic reticulum' in the cells they now investigated (the Huh7 human hepatoma cell line) (Stertz et al. 2006). Experimentally, Stertz et al. (2006) showed images of Huh7 cells with a cytoplasmic resticular meshwork distribution of HA-MxA. The customary markers for ER were of no avail, but an inhouse antibody towards syntaxin 17 was presented as a marker of the ER (see figure 3 below for the correct answer: that meshwork in Huh7 cells is of HA-MxA condensates adhering to intermediate filaments; Davis et al. 2019; Sehgal et al. 2020a).

The two articles - Accola et al. (2002) and Stertz et al. (2006) - have had a strong grip on the MxA field despite the inconclusive data presented. Hundreds of citations have referred to these two articles as evidence that MxA associates with smooth endoplasmic reticulum. Even the Kochs et al. (2002) article which showed 'membraneless' structures of MxA is repetitively misquoted in the Mx literature as having shown that MxA associates with the ER (see Sadler and Williams 2011 for one example).

Against this cumulative backdrop in the MxA field over the last 15 years, the author's lab stumbled on to observations that did not fit with the ER localization of MxA (Yuan and Sehgal 2016). Figure 1A shows examples of variably shaped and sized mesoscale structures in the cytoplasm of HEK293T cells expressing HA-MxA (using the expression construct provided by Stertz et al. 2006). Figure 1B shows that at higher magnification the HA-MxA structures were clearly distinct from the reticulon-4-containing endoplasmic reticulum (Yuan and Sehgal 2016; Davis et al. 2019). Numerous additional studies in the author's lab showed the divergence of MxA structures from the ER (summarized in Davis, et al. 2018; 2019). Thus, by the fall of 2016, we had concluded that there was little convincing experimental evidence that $\mathrm{MxA}$ associated with the ER. However, the paradigm in the field that 'MxA associates with the ER' based on Accola et al. (2002) and Stertz et al. (2006), was overwhelmingly and vigorously espoused (especially by numerous reviewers).

The formal observation by us in Dec 2017 using correlated light and electron microscopy (CLEM) methods that GFP-MxA structures in Huh7 cells were membraneless strengthened our inference ('MxA structures are not ER') (Davis et al. 2018 2019) (figure 2). These membraneless structures were associated with an extensive meshwork of intermediate filaments 

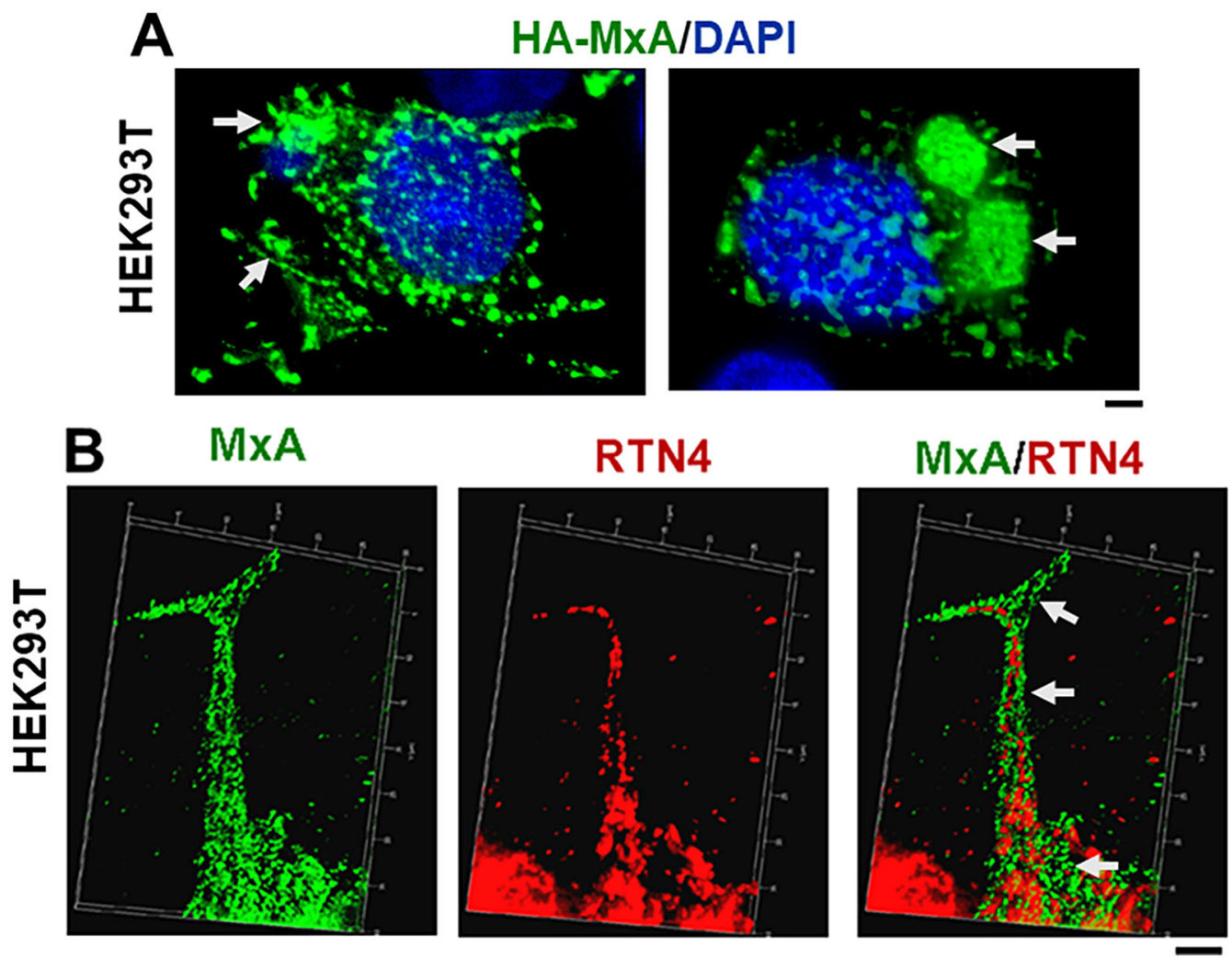

Figure 1. MxA forms variably sized and shaped mesoscale structures in the cytoplasm distinct from the endoplasmic reticulum (ER). Cultures of human HEK293T cells grown in $35 \mathrm{~mm}$ plates were transiently transfected the pHA-MxA expression vector (obtained from Stertz et al. 2006), fixed 1-2 days later, permeabilized using a digitonin-containing buffer, and the distribution of MxA and the ER structural protein reticulon-4 (RTN4) evaluated using immunofluorescence methods (imaged using an 100x oil immersion objective and z-stack data collection). Panel A, MxA structures in transiently transfected HEK293T cells (Scale bar $=10 \mu \mathrm{m}$ ). Arrows point to large compact mesoscale MxA-positive structures as observed in 25-35\% of transfected cells. Panel B, MxA granular structures were distinct from the RTN4-positive ER. Scale bar $=5 \mu \mathrm{m}$. Adapted from Yuan and Sehgal (2016) and Davis et al. (2019).

present in Huh7 hepatoma cells (figure 3; Davis et al. 20182019 and Sehgal et al. 2020a, b), thus accounting for the observations of Stertz et al. 2006. Indeed, thinsection electron microscopy studies using CLEM methods confirmed the juxtaposition of membraneless MxA structures adjacent to but distinct from intermediate filaments (Davis et al. 2018 2019) consistent with the immunofluorescence images in figure 3 . The further observation by us in June-July 2018 that exposure of Huh7 cells to hypotonic buffer led to the disassembly of GFP-MxA structures within 1-2 min, and that replenishment with isotonic culture medium led to rapid reassembly of the GFP-MxA structures (within 1 min) led us to an understanding of cytoplasmic MxA structures as metastable biomolecular membraneless condensates (figure 4; Davis et al. 2019). The GFPMxA condensates could be cycled through at least three such rounds of hypotonic disassembly and isotonic reassembly (figure 4). These GFP-MxA structures were also rapidly disassembled by 1,6 -hexanediol and had fluorescence recovery properties after photobleaching (FRAP) consistent with a gel-like consistency further confirming their identification as membraneless biomolecular condensates (Davis et al. 2019).

This paradigm shift has led us forward along two paths - the first to address questions about the condensate nature of human MxA structures in the cytoplasm and murine Mx1 structures in the nucleus (table 1), and the second to conduct extensive data mining of the Mx literature as part of the process of reinterpreting previous observations. As one example, we now infer that the membraneless human MxB structures juxtaposed to nuclear pores observed in 2004 by King et al. by electron microscopy also likely represent biomolecular condensates (table 1). 


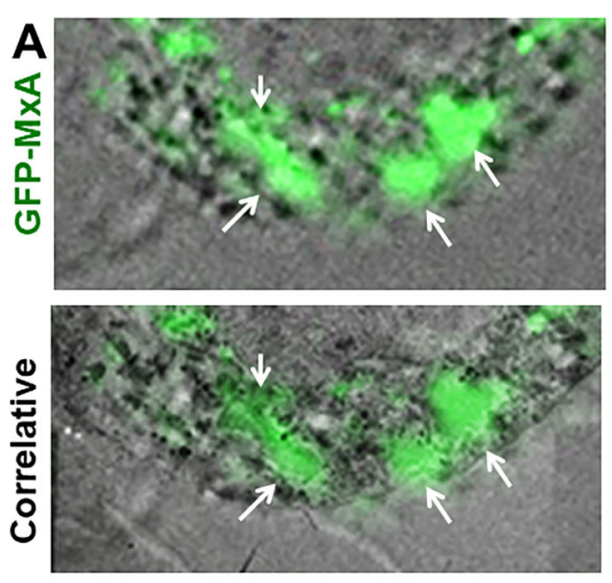

B
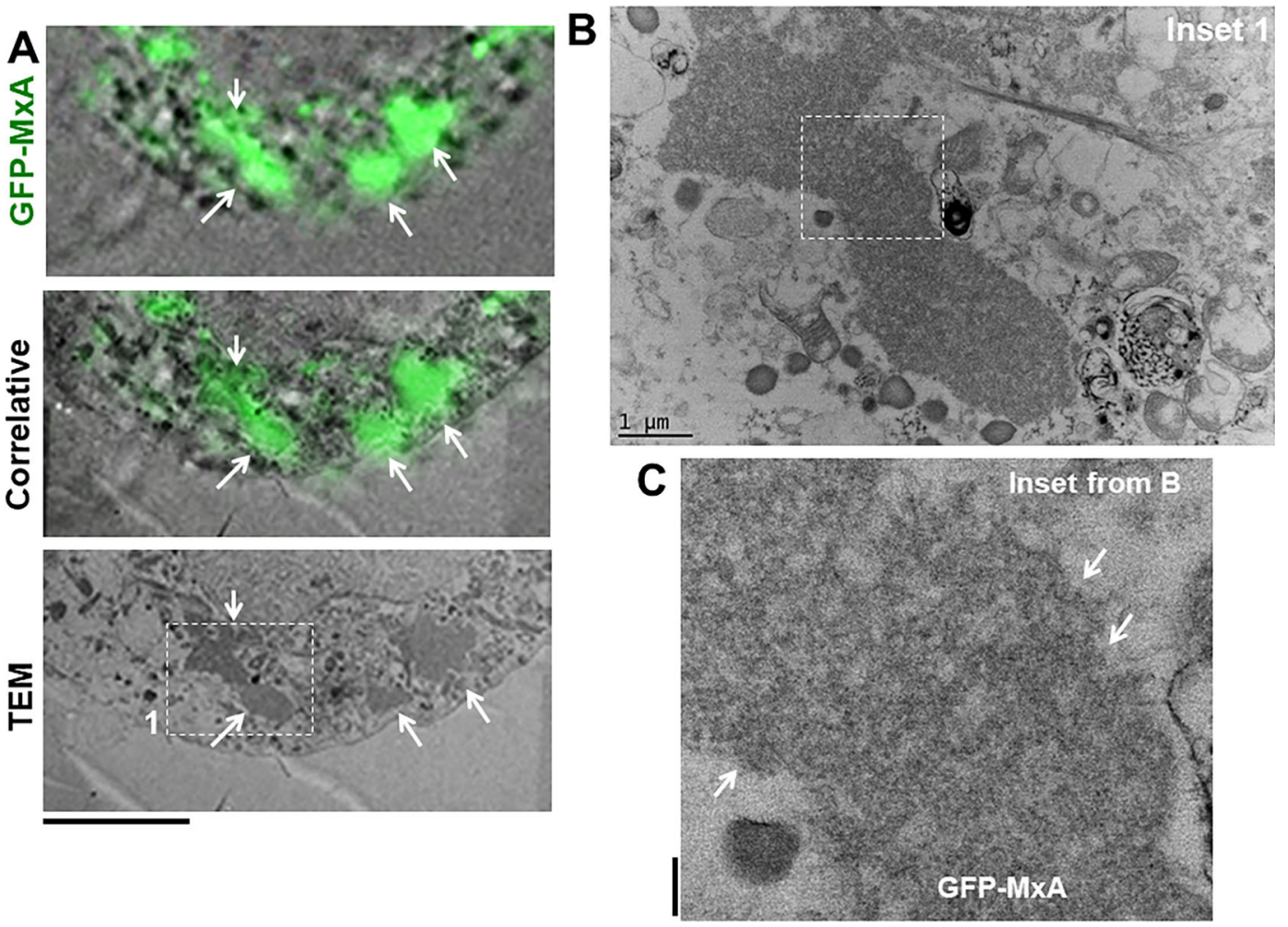

Figure 2. Correlated light and electron microscopy (CLEM) for identification of membraneless organelles. Huh7 cells plated sparsely in $35 \mathrm{~mm}$ gridded coverslip plates (MatTek) were transiently co-transfected with the pGFP-MxA vector. Two days later the cultures were fixed with $4 \%$ paraformaldehyde for $1 \mathrm{~h}$ at $4^{0}$. Confocal imaging was carried out using a tiling protocol to identify the location of specific cells with GFP-MxA structures on the marked grid. The cultures were then further fixed, embedded and the previously identified grid locations used for serial thin-section EM (TEM). The tiled light microscopy data were correlated with the tiled EM data to identify the ultrastructure of the GFP-fluorescent structures (arrows in Panel A). Scale bar $=5 \mu \mathrm{m}$. Panel B shows a higher magnification image of inset 1 indicated in bottom image in Panel A. Scale bar $=1 \mu \mathrm{m}$. Panel $\mathrm{C}$ shows a higher magnification TEM images of the boxed portion in Panel B. Arrows indicate the absence of an enveloping membrane. Scale bar $=200 \mathrm{~nm}$. Figure adapted from Davis et al. (2019).

Clearly at this time, there are many unanswered questions. The reader is referred to previous detailed reviews on the biology of Mx proteins in the context of innate immunity (Haller et al. 2015 2018). At this time, it would be premature for us tie aspects of the cell biology of Mx condensates (assembly/disassembly and metastability) to the broader biology of $\mathrm{Mx}$ proteins (antiviral activity against different viruses). Also, at this time, we are unaware of the identification of other dynamin-family large GTPases or other IFN-induced proteins that form biomolecular condensates (we are sure that these will come to be identified soon). The focus of this overview is to provide an interim summary of our data which helped identify cytoplasmic MxA and nuclear Mx1 structures as biomolecular condensates. It should be apparent from this Introduction that we are the only research group in the Mx field that has moved in this direction at this time.

\section{Ubiquity of biomolecular condensates and phase-separated membraneless organelles (MLOs)}

As a general comment, in addition to various membrane-bound subcellular compartments, the eukaryotic cell contains organized membraneless biomolecular condensates of proteins and nucleic acids which form functional organelles (Mitrea and Kriwacki 2016; Banani et al. 2017; Shin and Brangwynne 2017; Alberti 2017; Gomes and Shorter 2019; Alberti et al. 2019; Sehgal et al. 2020a). Examples include the nucleolus, the nucleoporin granules, nuclear speckles and paraspeckles, nuclear promyelocytic leukemia (PML) bodies, nuclear Cajal bodies, cytoplasmic processing $(\mathrm{P})$ bodies, germinal $\mathrm{P}$ bodies, Balbiani bodies, Negri bodies, stress granules, translation promoting TPA-inducible sequence $11 \mathrm{~b}$ (TIS) granules, cytoplasmic and nuclear viral replication and maturation 

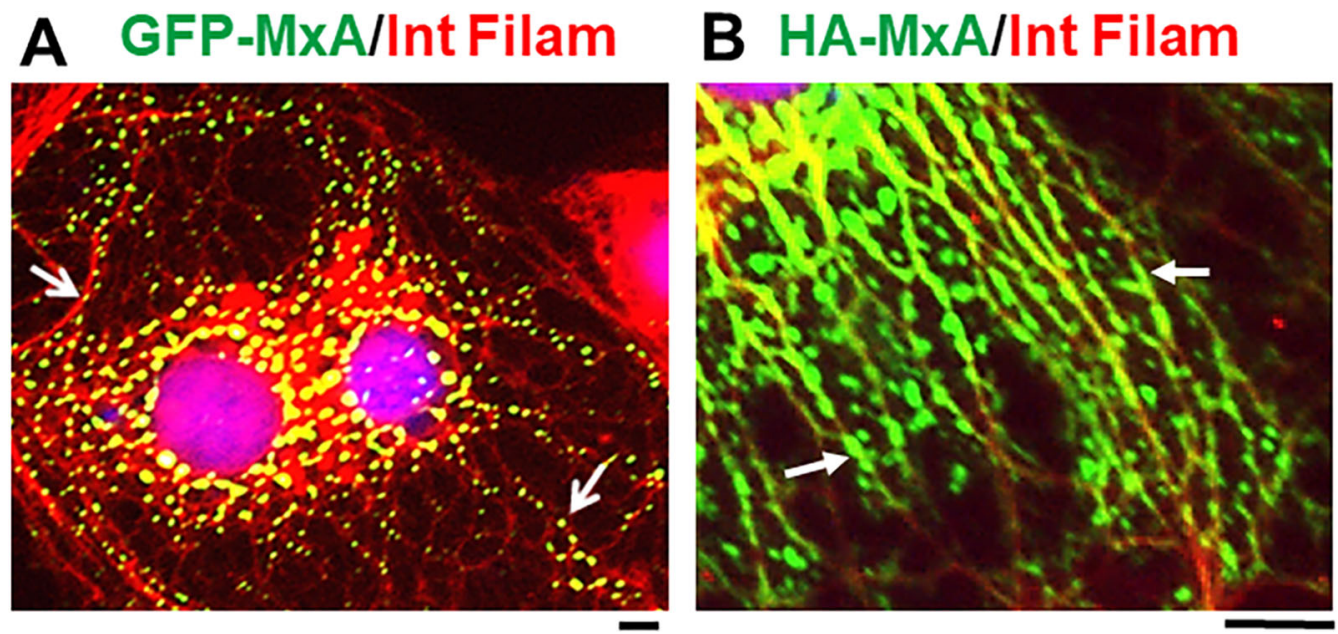

Figure 3. HuMxA condensates in Huh7 hepatoma cells often use intermediate filaments as scaffolding. Human hepatoma Huh7 cells cultured in $35 \mathrm{~mm}$ plates were transiently transfected with expression vector for GFP-MxA (Panel A) or HA-MxA (Panel B). Two days later the cultures were fixed and imaged for GFP-MxA (Panel A, by fluorescence in green), HA-MxA (Panel B, by immunofluorescence using anti-HA mAb in green), and intermediate filaments (Panels $\mathbf{A}$ and $\mathbf{B}$, using antigiantin pAb in red; see Sehgal et al. 2020b for validation). White arrows point to spherical GFP-MxA structures and streaks of HA-MxA adhering to intermediate filaments in Panels $\mathbf{A}$ and $\mathbf{B}$ respectively. Scale bars $=5 \mu \mathrm{m}$. Adapted from Davis et al. (2019) and Sehgal et al. (2020a).

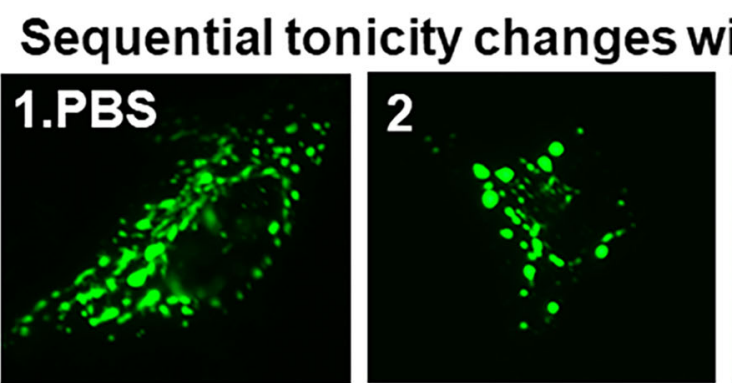

ELB \pm Sucrose > +

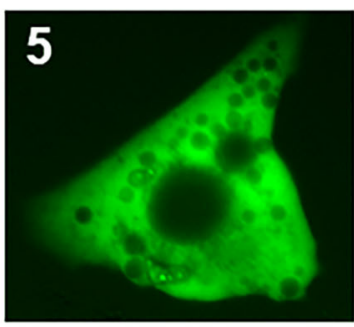

-

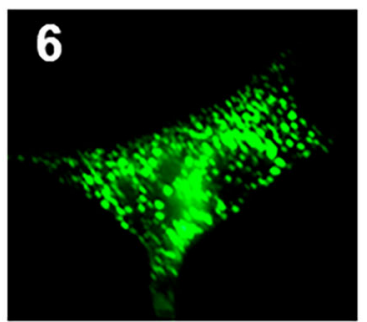

$+$

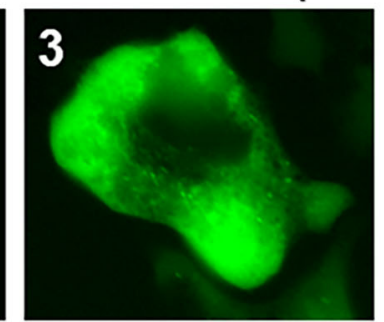

-

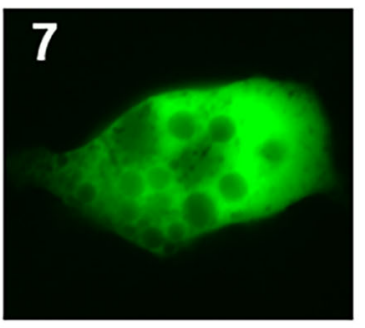

-

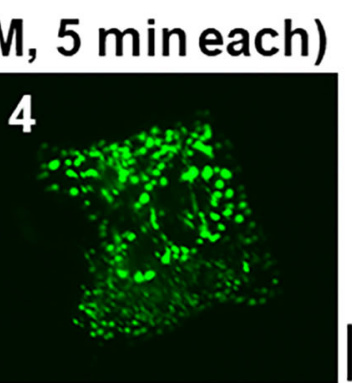

$+$

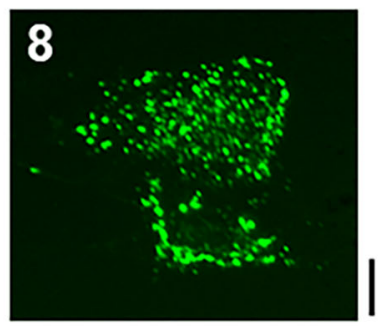

$+$

Figure 4. Repetitive tonicity-driven disassembly and reassembly of GFP-MxA condensates. A culture of Huh7 cells in 35 mm plate transiently expressing GFP-MxA kept continuously at $37{ }^{\circ} \mathrm{C}$ was sequentially imaged in 5 min steps in the indicated media [warm isotonic phosphate buffered saline (PBS) or warm hypotonic erythrocyte lysis buffer (ELB) without or with supplementation with $0.3 \mathrm{M}$ sucrose indicated $(-$ or +$)$ ]. Images $1-8$ illustrate representative examples of cells at each stage in the same culture (out of 10-20 images per step). Scale bars $=10 \mu \mathrm{m}$. Figure adapted from Davis et al. (2019).

complexes, and several more recent discoveries such as condensates of synapsin, of the DNA sensor protein cyclic GMP-AMP synthase (cGAS) in the cytoplasm, the cytoplasmic antiviral proteins human MxA, the nuclear antiviral protein murine $\mathrm{Mxl}$, and active transcription- and splicing-associated condensates in the nucleus. Overall, these condensates have liquid-like internal properties and alter shape and consistency (are 'metastable') changing from liquid-like to a gel and/or to filaments and even undergo disassembly/reassembly 
cycles commensurate with the cellular environment (temperature, ionic conditions and tonicity, physical deformation or cytoplasmic 'crowding'), and the incorporation of additional proteins, RNA or DNA molecules or posttranslational modifications (Mitrea and Kriwacki 2016; Banani et al. 2017; Shin and Brangwynne 2017; Alberti 2017; Alberti et al. 2019; Gomes and Shorter 2019; Sehgal 2019; Davis et al. 2018 2019; Sehgal et al. 2020a, b; Du and Chen 2018; Milovanovic et al. 2018; Delarue et al. 2018; Boke et al. 2016; Sabari et al. 2018; Ditlev et al. 2018; Rai et al. 2018; Boeynaems et al. 2019; Ma and Mayr 2018). Indeed, DNA and RNA molecules specifically participate in the assembly of such cytoplasmic and nuclear condensates and in their function (Mitrea and Kriwacki 2016; Banani et al. 2017; Shin and Brangwynne 2017; Alberti 2017; Alberti et al. 2019; Gomes and Shorter 2019; Du and Chen 2018; Milovanovic et al. 2018; Delarue et al. 2018; Boke et al. 2016; Sabari et al. 2018; Ditlev et al. 2018; Ma and Mayr 2018). Moreover, in many instances the dynamic assembly, disassembly and reassembly of such condensates within a cell compartment is regulated during differentiation, cell cycle, stress and under pathological conditions (reviewed in Rai et al. 2018; Gomes and Shorter 2019; Sehgal 2019; Sehgal et al. 2020a). Collectively, such condensates form a hierarchy of distinct membraneless organelles (MLOs) in the cytoplasm and nucleus which serve distinct cellular functions.

\section{Different Mx GTPase protein family members form biomolecular condensates in different subcellular compartments}

The Mx proteins are now established as the major antiviral effectors of Type I (IFN- $\alpha / \beta)$ and Type III (IFN$\lambda$ ), but not Type II (IFN- $\gamma$ ) interferons (Kochs et al. 2002; Haller and Kochs 2002; Haller et al. 2007 2015; Verhelst et al. 2013) (figure 5). The Mx proteins are now recognized to inhibit a broad spectrum of RNA- and DNAcontaining viruses (Haller and Kochs 2002; Haller et al. 2015 2018; Verhelst et al. 2013). Mx proteins are large dynamin-family GTPases of size $60-70 \mathrm{kDa}$ which readily multimerize into rings and filaments and contain intrinsically disordered regions (e.g. the L4 loop) which contribute to multimerization (figure 5) (Haller et al. 2015). There are two human Mx proteins (MxA and $\mathrm{MxB})$ and two murine $\mathrm{Mx}$ proteins $(\mathrm{Mx} 1$ and $\mathrm{Mx} 2)$ of different antiviral activities and different subcellular localizations - cytoplasmic vs. nuclear (table 1, figure 5). To clarify the nomenclature of the Mx protein family we adopt the gene lineage tracing presented by Busnadiego et al. (2014) and Haller et al. (2015) as follows: most mammalian $\mathrm{Mx}$ proteins are formed from two distinct gene lineages $(M x A$ or $M x B)$ that arose from an ancient duplication event. Thus, humans have two Mx proteins MxA and MxB (some investigators call these as human Mx1 and human Mx2 respectively although the terms $\mathrm{Mx} 1$ and $\mathrm{Mx} 2$ are correctly reserved for murine $\mathrm{Mx}$ proteins). Although mice also have two Mx genes - Mxl and $M x 2$, both these are paralogous members of the human $M x A$ lineage. Thus, human $M x B$ (which is also called human $M x 2$ by some investigators) and murine Mx2 are not orthologous. We use the terms MxA or HuMxA for the human protein, and Mx1 or MuMx1 for the orthologous murine protein. Parenthetically, rats have three Mx proteins Mx1, Mx2 and Mx3 (Haller et al. 2015). Rat Mx1 and rat Mx2 are orthologs of human MxA, have antiviral activity and form nuclear and cytoplasmic granules respectively, while rat $\mathrm{Mx} 3$ is diffuse in the cytoplasm and lacks antiviral activity.

Human MxA forms cytoplasmic structures while murine Mx1 mainly forms nuclear bodies (table 1; figure 6; Haller and Kochs 2002; Kochs et al. 2002; Engelhardt et al. 2004; Haller et al. 2015; Sehgal et al. 2020b). The molecular mechanisms of the Mx antiviral effects are incompletely understood; these effects include inhibition of early and late viral transcription and viral replication, as well as effects at the level of the transit of viral components through the nuclear pore (Haller and Kochs 2002; Verhelst et al. 2013; Haller et al. 2015; Steiner and Pavlovic 2020). It is only in the last 3 years that we recognized that human MxA formed metastable membraneless biomolecular condensates in the intact-cell cytoplasm, and only in the last year that murine $\mathrm{Mx} 1$ formed biomolecular condensates mainly in the nucleus, but also in the cytoplasm in some cells (figure 6; reviewed in Sehgal et al. 2020a, b). Both the cytoplasmic HuMxA bodies and nuclear MuMx1 bodies comprised phase-separated membraneless organelles (MLOs) with a gel-like internal consistency (as tested using fluorescence recovery after photobleaching (FRAP) methods) (Davis et al. 2019; Sehgal et al. 2020a, b). Additionally, HuMxB forms cytoplasmic membraneless structures juxtaposed to the cytoplasmic side of nuclear pores (Table 1) (King et al. 2004; Goujon et al. 2013; reviewed in Sehgal et al. 2020b; Steiner and Pavlovic 2020).

Remarkably, it is also now recognized that replication/maturation of many viruses also involves phaseseparated liquid droplets [e.g. vesicular stomatitis (VSV), rabies (Negri bodies), influenza A, Ebola, measles, Epstein-Barr, and even SARS-CoV-2 viruses] (reviewed 


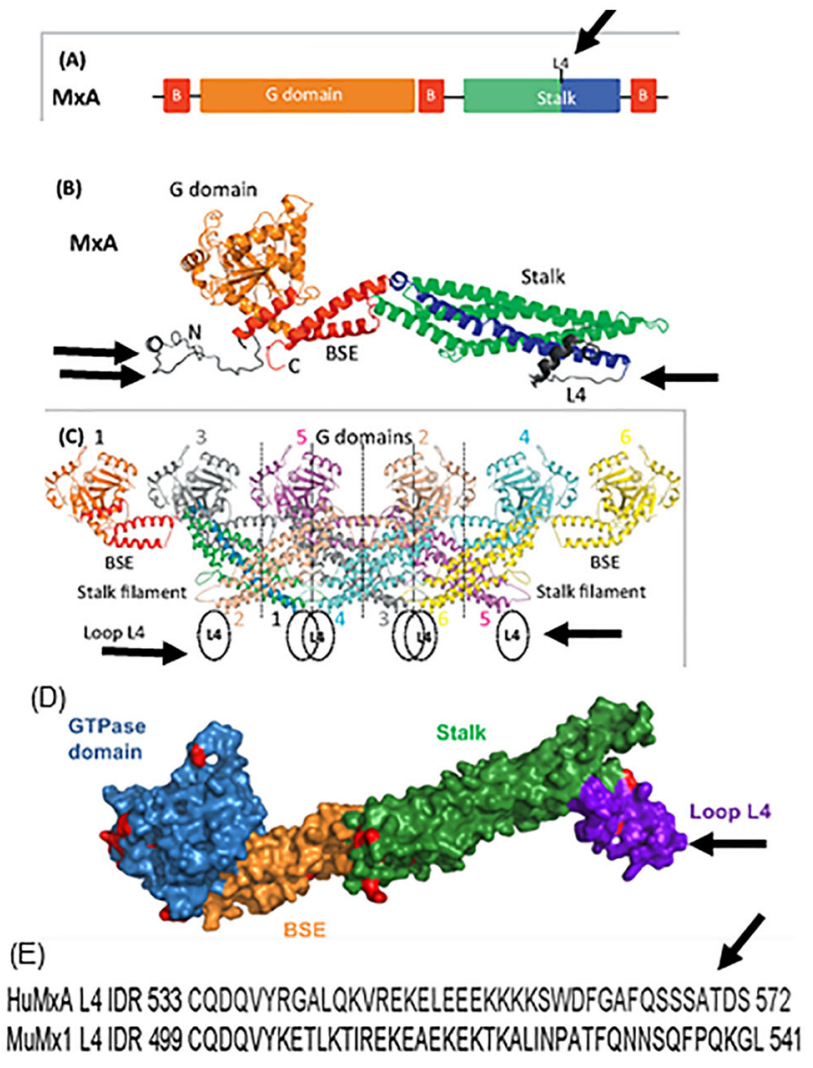

Figure 5. Key features of the structure of MxA monomers and oligomers. Panels $\mathbf{A}$ and $\mathbf{B}$, schematic and 3D structure of respective color-coded domains in MxA GTPase monomers. The GTPase G domain forms the head group, the Stalk consists of three $\alpha$ helices which help drive oligomerization, while the BSE hinge region is made up of $\alpha$-helices from three different portions of the MxA sequence (labelled ' $\mathrm{B}$ ' in Panel A. L4 (single arrow) represents the polylysine intrinsically disordered domain (IDR), while the double arrow points to a disordered domain at the $\mathrm{N}$ terminus. Panel C, Structure of MxA oligomers (linear or ring shaped) formed in cell-free assays driven by the stalk filaments and by the L4 loops (arrow). Panel D, Space-filling model of the MxA monomer highlighting the exposed location of the L4 IDR region (arrow). Panel E, Comparison of the sequences of the IDR loop L4 in HuMxA and MuMx1. Modified from Haller et al. (2015) with permission.

in Sehgal et al. 2020a, b). It is noteworthy that MxA cytoplasmic condensates in virus infected cells incorporate respective viral nucleocapsid proteins (table 1) (Kochs et al. 2002; Davis et al. 2019; reviewed in Sehgal et al. 2020a, b). Overall, human MxA forms disparate membraneless structures solely in the cytoplasm and has antiviral activity towards several RNA- and DNA-containing viruses including orthomyxo- and rhabdoviruses (Haller et al. 2015; Sehgal et al. 2020a, b). Human MxB is mainly associated with the cytoplasmic side of nuclear pores (through an N-terminal nuclear localization signal,
NLS) and additional cytoplasmic membraneless structures. Full-length $\mathrm{MxB}$ has antiviral activity against HIV and other lentiviruses, and herpesviruses by blocking entry of viral components into the nucleus, but not against influenza A virus (FLUAV) (King et al. 2004; Goujon et al. 2013; reviewed in Haller et al. 2015 and in Steiner and Pavlovic 2020). However, ectopic expression of genetically modified $\mathrm{MxB}$ targeting it to the cytoplasm (using the $\mathrm{N}$-terminal 43 amino acid segment from MxA) or nucleus (using the T-antigen NLS) renders $\mathrm{MxB}$ antiviral towards FLUAV (Steiner and Pavlovic 2020).

Murine Mx1 is mainly in nuclear bodies (it has a 'weak' C-terminal NLS, Zürcher et al. 1992a, b, c), while murine Mx2 is mainly in cytoplasmic structures (Zürcher et al. 1992c; Haller et al. 2015). In terms of antiviral activity, the nuclear murine Mx1 has antiviral activity towards influenza virus (FLUAV) (which has an obligate nuclear transcription step) but not vesicular stomatitis virus (VSV) (replicates entirely in the cytoplasm), while the cytoplasmic granular murine $\mathrm{Mx} 2$ has antiviral activity towards VSV but not FLUAV (Haller et al. 2015; table 1). Curiously, the nuclear-predominant rat Mx1 has antiviral activity towards both FLUAV and VSV, while the cytoplasmic-predominant rat $\mathrm{Mx} 2$ is antiviral towards VSV only (Verhelst et al. 2013; Haller et al. 2015). Rat Mx3, which is mainly dispersed in the cytoplasm, has little apparent antiviral activity (Verhelst et al. 2013; Haller et al. 2015). Although murine Mx1 artificially deleted of its C-terminal NLS is cytoplasmic it remains inactive against VSV (Zürcher et al. 1992a, 1992b). In contrast, wild-type GFP-MuMx1 expressed in human hepatoma Huh7 cells associates with cytoplasmic intermediate filaments and now shows antiviral activity against VSV (Sehgal et al. 2020b, table 1 and figures 7 and 8 below). Thus, the different subcellular localizations of MLOs of different $\mathrm{Mx}$ proteins contribute to their respective antiviral activities. Our overall focus is to understand the formation, dynamics and function of different antiviral Mx biomolecular condensates. The objective is to move the field of antiviral Mx proteins into the realm of phaseseparated membraneless organelles (MLOs) and biomolecular condensates, and set the stage for detailed proteomics studies in the future of the composition of $\mathrm{Mx}$ condensates.

\section{Metastability of biomolecular condensates of Mx proteins}

Cytoplasmic human MxA condensates and nuclear murine Mx1 condensates (figure 6) comprised dramatically metastable (shape-changing) phase-separated 

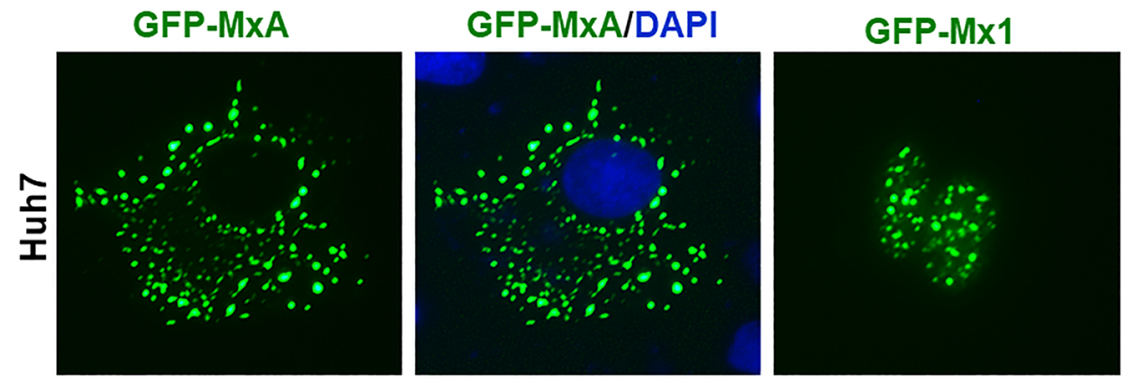

GFP-Mx1/DAPI
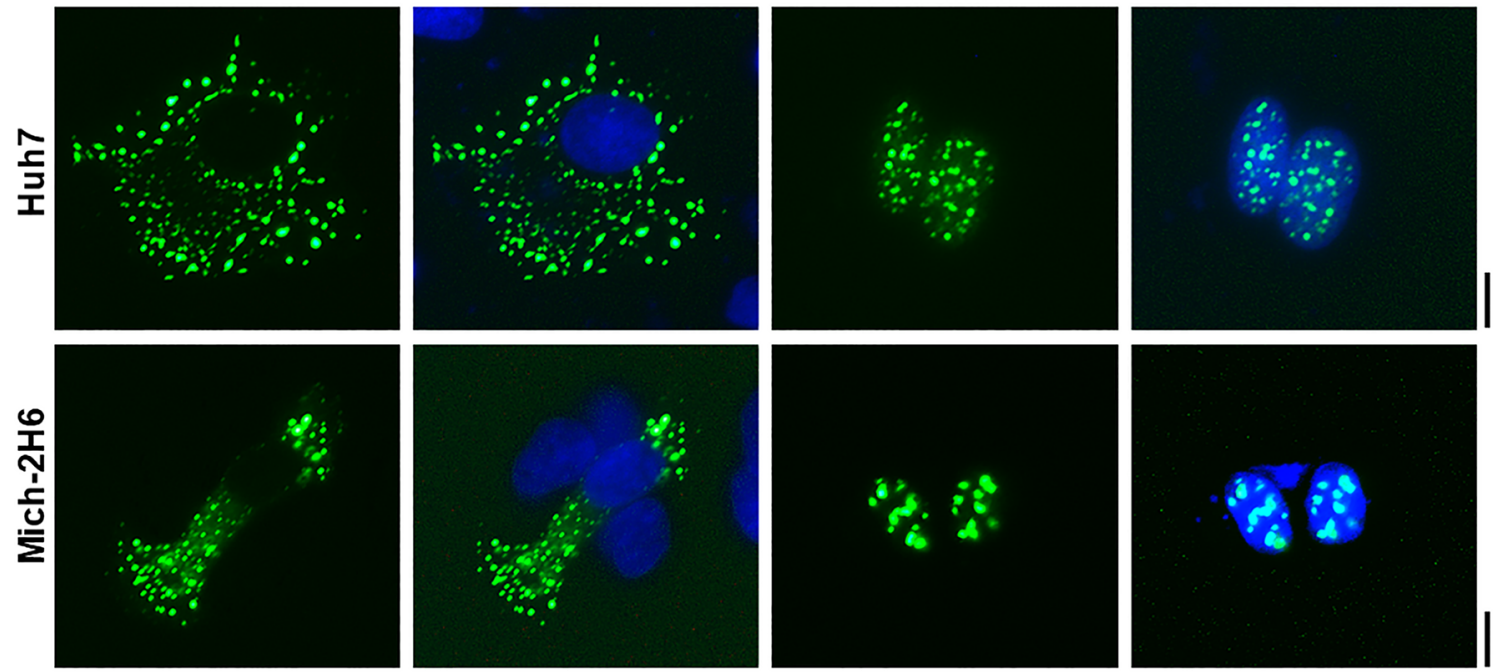

Cytoplasmic

Nuclear

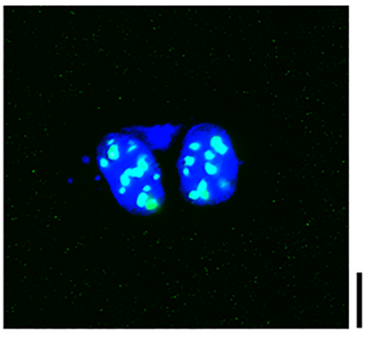

Figure 6. Comparison of subcellular localization of human GFP-MxA with murine GFP-Mx1 in two different cell lines. Cultures of Huh7 hepatoma and Mich-2H6 melanoma cells in $35 \mathrm{~mm}$ plates were transiently transfected with expression vectors for human GFP-MxA or murine GFP-Mx1, fixed using 4\% paraformaldehyde 2 days later, additionally stained with DAPI to visualize the nuclei, and imaged using two-color fluorescence. The figure illustrates representative cells. None of the cells transfected with human GFP-MxA vector showed any MxA in nuclei; 70-80\% of Huh7 cells transfected with GFP-Mx1 showed only nuclear Mx1 (for cells with cytoplasmic GFP-Mx1 structures see figures 7 and 8); almost all of Mich-2H6 cells transfected with GFP-Mx1 showed only nuclear GFP-Mx1 bodies. Scale bars $=10 \mu \mathrm{m}$. From Sehgal et al. (2020b).

MLOs (Davis et al. 2018 2019; Sehgal et al. 2020a, b; table 1). These GFP-MxA and GFP-Mx1 condensates were disassembled by 1,6-hexanediol (and were thus liquid-like phase separated structures) (Davis et al. 2019). Disassembly of nuclear GFP-Mx1 bodies by hexanediol was than that of cytoplasmic GFP-MxA bodies (Sehgal et al. 2020b). Both cytoplasmic GFPMxA and nuclear GFP-Mx1 condensates showed a gellike internal consistency in FRAP assays with approximately $18-20 \%$ internal mobility) (Davis et al. 2019; Sehgal et al. 2020b). Importantly, IFN- $\alpha$-induced endogenous MxA in human cells also formed cytoplasmic condensates (Davis et al. 2018 2019; Sehgal et al. 2020a).

As another aspect of their metastability, both cytoplasmic GFP-MxA condensates and nuclear GFP-Mx1 condensates were disassembled by exposing cells to hypotonic buffer (ELB), and then reassembled upon shifting cells to isotonic buffer (figure 4; Davis et al. 2019; Sehgal et al. 2020b). This hypotonicity-driven disassembly/metastability is reminiscent of conditions of cells with intracellular edema. This metastability was also observed for GFP-Mx1 nuclear condensates in homologous murine cells (NIH 3T3) (Sehgal et al. 2020b). We interpret hypotonic disassembly/isotonic reassembly as resulting from rapid cytoplasmic uncrowding and recrowding mechanisms (i.e. responsive to cytoplasmic swelling and its reversal) (Davis et al. 2019; Sehgal et al. 2020a, b).

Live-cell imaging confirmed that spherical cytoplasmic MxA condensates underwent homotypic fusion (Davis et al. 2019). There was further metastability in terms of a spherical to filamentous change when cells were exposed to physical compression and/ or hypoxia (by placement on a coverslip on live cells), nitric oxide scavenging (by c-PTIO) or inhibition of GTPase activity (by dynasore) (Davis et al. 2019). The unanswered question at the moment is whether these changes influence antiviral activity.

\section{Heterogeneity of condensates of Mx proteins}

As with stress granules (Ditlev et al. 2018; Shiina 2019), there is structural heterogeneity in cytoplasmic MxA condensates, especially the presence of a detergent (saponin) resistant core in some condensates (Davis et al. 2019). MxA condensates disassembled upon saponin permeabilization of the plasma membrane of cells consistent with an 'uncrowding' mechanism regulating MxA condensate structure. Additionally, $20-30 \%$ of cells retained saponin- 


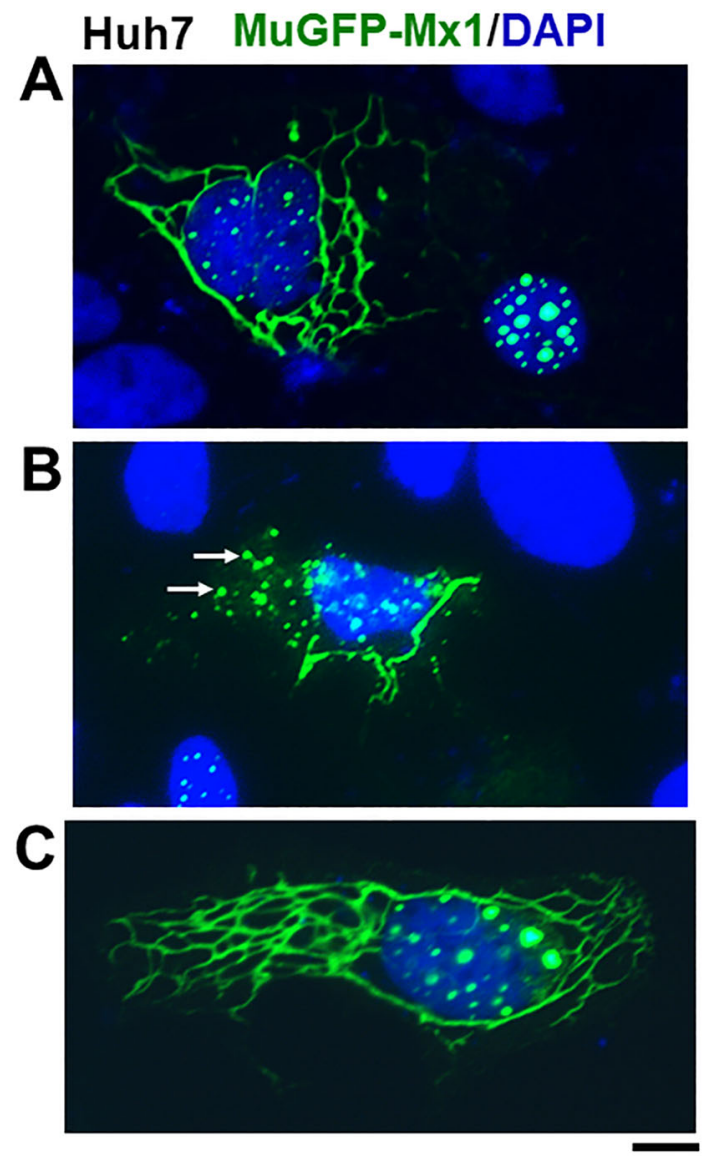

Figure 7. Spherical nuclear, spherical cytoplasmic and filamentous cytoplasmic condensates of murine GFP-Mx1. Cultures of Huh7 cells in $35 \mathrm{~mm}$ plates were transiently transfected with the expression vector for murine GFP-Mx1. Two days later the cultures were fixed using $4 \%$ paraformaldehyde, permeabilized using the $0.05 \%$ Triton buffer, and stained with DAPI to visualize the nuclei, and imaged using two-color fluorescence. Approximately $20-30 \%$ of transfected cells showed cytoplasmic murine GFP-Mx1 as shown in Panels A, B and C. Panel A shows two adjacent cells - one showing GFP-Mx1 exclusively in nuclear bodies and one cell with GFP-Mx1 present extensively in cytoplasmic filaments and cytoplasmic structures. Panel B shows adjacent cells - one with extensive cytoplasmic filaments and cytoplasmic bodies (arrows) of GFP-Mx1 and the other showing a nucleus with only nuclear GFP-Mx1 bodies (at lower edge). Panel $\mathrm{C}$ shows a cell with both nuclear GFP-Mx1 bodies and cytoplasmic filaments. Modified from Sehgal et al. (2020b).

resistant 'cores' of GFP-MxA condensates (Davis et al. 2019). Thus, in future studies, we shall follow the paradigm established in studies of stress granule components (Banani et al. 2017; Ditlev et al. 2018; Alberti et al. 2019; Shiina 2019), by first identifying components of MxA condensate cores, before exploring the more transient association of other client proteins with these structures.

While human GFP-MxA structures were exclusively cytoplasmic, and murine GFP-Mx1 structures mainly nuclear (as in figure 6), a subset of cells (in human Huh7 and murine NIH3T3 cell lines) transiently expressing MuGFP-Mx1 showed accumulation of this protein in cytoplasmic bodies and filaments (figure 7) (Sehgal et al. 2020b). This formation of cytoplasmic GFP-Mx1 structures was not a consequence of overexpression of the recombinant protein but occurred even when there was only low level of expression of GFP-Mx1 (Sehgal et al. 2020b). In Huh7 cells, these cytoplasmic filaments of GFP-Mx1 consisted of vimentin-positive intermediate filaments (Sehgal et al. 2020b). The possibility that, in contrast to GFPHuMxA structures which lie adjacent to intermediate filaments (figure 3A), the GFP-MuMx1 might be part of the structure of intermediate filaments remains open.

This heterogeneity of subcellular location of MuGFP-Mx1 condensates was reflected in differences in antiviral properties (Sehgal et al. 2020b). Using single-cell-based assays for antiviral activity towards $\mathrm{VSV}$, on the one hand we were able to confirm that cytoplasmic human MxA was antiviral towards VSV, but that nuclear murine Mx1 was not (figure 8). On the other hand, cells exhibiting cytoplasmic filamentous wild-type MuGFP-Mx1 had an antiviral effect against VSV (figure 8). These data emphasize the occurrence of a broader antiviral spectrum of Mx proteins in different cell types based upon the subcellular cytoplasmic compartment in which the respective Mx proteins form condensates (Haller et al. 2015; Sehgal et al. 2020b; also see Steiner and Pavlovic 2020 for MxB).

\section{Comments}

Overall, our new data show that (a) both human MxA and murine $\mathrm{Mx} 1$ give rise to metastable phase-separated biomolecular condensates, albeit in different subcellular compartments (cytoplasm vs. nucleus respectively), (b) when in the cytoplasm, both human MxA and murine Mx1 can associate with intermediate filaments, and (c) both human MxA and murine Mx1 when cytoplasmic exhibit an antiviral activity towards VSV - a rhabdovirus which replicates and matures entirely in the cytoplasm (table 1). The data showing the close relationship between membraneless $\mathrm{MxB}$ structures and nucleoporins on the cytoplasmic face of nuclear pores and the involvement of $\mathrm{MxB}$ in regulating cargo transit through the pore channel (King 


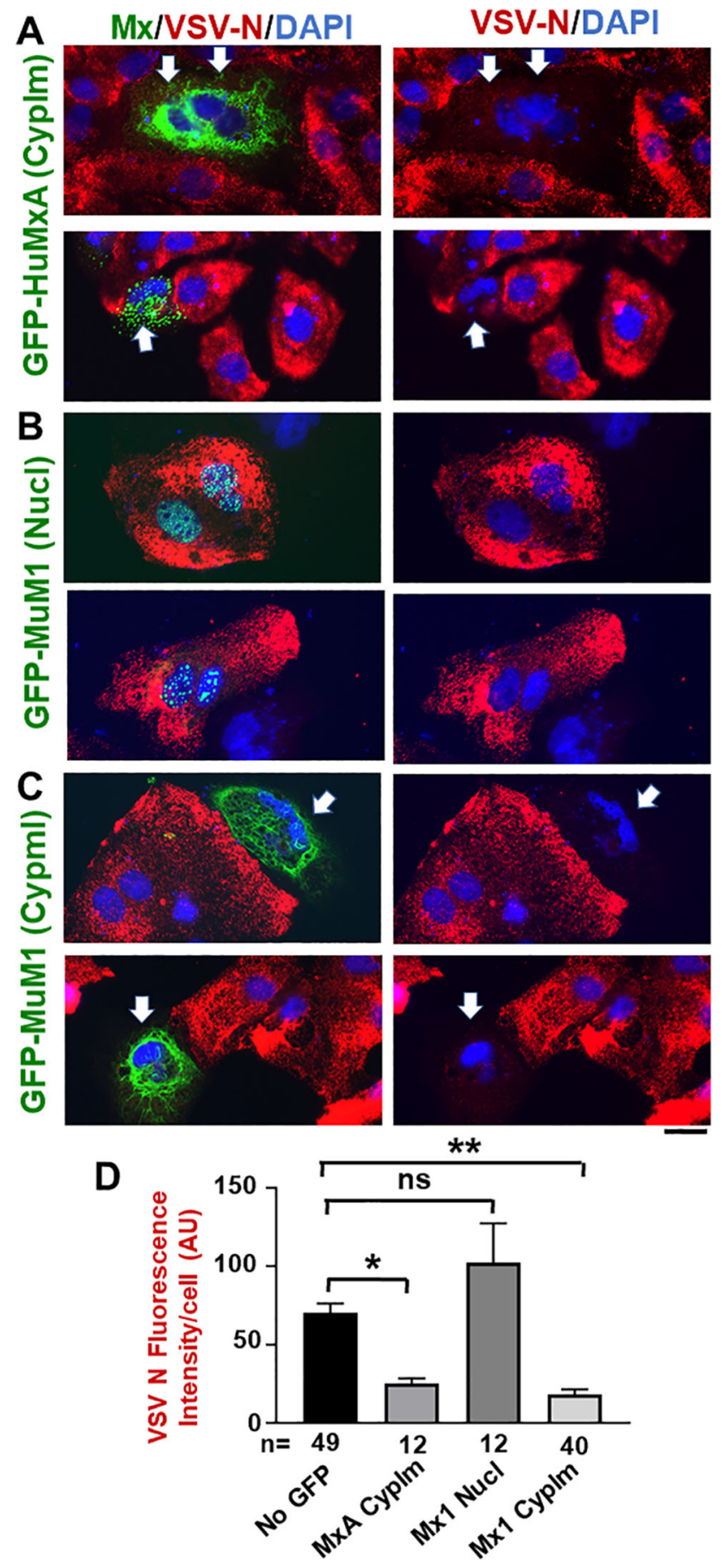

et al. 2004; Goujon et al. 2013; reviewed in Steiner and Pavlovic 2020) allow us to now suggest that MxB structures at and near nuclear pores also represent phase-separated biomolecular condensates (table 1; Sehgal et al. 2020b).

Murine Mx1 typically shows antiviral activity towards influenza A virus (FLUAV) and other orthomyxoviruses which require a nuclear step in their replication, but not towards rhabdoviruses such as
4 Figure 8. Differences in subcellular localization of MuGFP-Mx1 condesnates (in cytoplasm vs. nucleus) affects antiviral activity towards VSV. Huh7 cells (approx. $2 \times 10^{5}$ ) per $35 \mathrm{~mm}$ plate, transfected with the GFP-HuMxA (as a positive control) or GFP-MuMx 1 expression vectors 2 days earlier, were replenished with $0.25 \mathrm{ml}$ serum-free Eagle's medium and then $20 \mu \mathrm{l}$ of a concentrated VSV stock of the wt Orsay strain added (corresponding to multiplicity of infection $>10$ plaque forming units/cell). The plates were rocked every $15 \mathrm{~min}$ for $1 \mathrm{hr}$ followed by addition of $1 \mathrm{ml}$ of full culture medium. The cultures were fixed with $4 \%$ paraformaldehyde at $4 \mathrm{hr}$ after the start of the VSV infection and the extent of VSV N protein expression in individual cells evaluated using immunofluorescence methods (using the mouse anti-N mAb) and Image $J$ for quantitation. Panels $\mathbf{A}, \mathbf{B}$ and $\mathbf{C}$ illustrate representative cells showing the absence of any GFP, or the appearance of cytoplasmic GFPHuMxA, nuclear GFP-MuMx1 or cytoplasmic GFP-MuMx1 and the corresponding level of expression of viral $\mathrm{N}$ protein (thick arrows point to cells displaying an antiviral effect). All scale bars $=20 \mu \mathrm{m}$. Panel $\mathbf{D}$, enumerates $\mathrm{N}$ protein expression in various classes of cells shown in Panels $\mathbf{A}$, $\mathbf{B}$ and $\mathbf{C}$ imaged at identical exposure settings and expressed in arbitrary fluorescence units (AU) per cell. $n=$ number of cells evaluated per group in this experiment (for this evaluation cells with only cytoplasmic Mx1 were combined with cells with both cytoplasmic and nuclear Mx1); vertical columns depict Mean \pm SE. Statistical significance was evaluated using ANOVA (Kruskal-Wallis with Dunn's posttest for multiple comparisons); ${ }^{*} P<0.01$; ** $P<0.001$; ns, not significant $(P>0.05)$. Adapted from Sehgal et al. (2020b).

VSV which replicate entirely in the cytoplasm (Verhelst et al. 2013; Haller et al. 2015) (table 1). In contrast, functional murine $\mathrm{Mx} 2$ (isolated from feral mice) was observed to display an antiviral activity towards VSV but not FLUAV (Verhelst et al. 2013; Haller et al. 2015). Commensurately, while murine Mx1 was observed to be in nuclear bodies, murine Mx2 was observed to be mainly in granular cytoplasmic structures (Haller et al. 2015), which might perhaps also represent biomolecular condensates (table 1). Moreover, we observed that a subset of cells expressing murine GFP-Mx1 express this protein in cytoplasmic bodies and filaments. Using single-cellbased assays for VSV replication we discovered that cells that expressed cytoplasmic wild-type GFP-Mx1, but not those exhibiting only nuclear GFP-Mx1 condensates, displayed an antiviral effect against this virus - a virus which replicates solely in the cytoplasm.

Mutational studies of human MxA show that the GTPase activity is required for most of its antiviral 
activity (except that against hepatitis B virus) (Haller et al. 2015; Dick et al. 2015; Nigg and Pavlovic 2015; Steiner and Pavlovic 2020). Data in the literature also reveal that MxA mutants lacking GTPase activity can still form cytoplasmic condensates (Dick et al. 2015; Nigg and Pavlovic 2015). Mutations that cause dispersal of MxA in the cytoplasm (e.g. the D250N mutant) lacked antiviral activity (Dick et al. 2015; Steiner and Pavlovic 2020). The R645 point mutant of MxA, which was in larger cytoplasmic granules, had the unusual property of inhibiting FLUAV but not VSV, even though the wt MxA showed antiviral activity towards both viruses (Zürcher et al. 1992b). A mutational analysis of rat Mx2 showed that mutants that formed 'granular' cytoplasmic structures exhibited antiviral activity towards VSV, while those that were 'diffuse' in the cytoplasm did not (Zürcher et al. 1992c). These data suggest that condensate formation may be important but not sufficient for the antiviral activity of Mx proteins.

The hypotonicity-driven disassembly of Mx protein condensates in live cells (figure 4; Davis et al. 2019; Sehgal et al. 2020b) highlight an unusual aspect of $\mathrm{Mx}$ protein chemistry. The biochemical basis for this hypotonicity driven disassembly may reflect the effect of cytoplasmic 'crowding' on higher-order protein structure or rapid changes due to hypotonicity-triggered post-translational modifications.

At this time there are many unanswered questions related to the biochemical mechanisms involved in the formation and metastability of Mx condensates in the cell cytoplasm and the nucleus. The structural contributions of intrinsically disordered domains (IDRs) in $\mathrm{Mx}$ proteins (the L4 loop and the $\mathrm{N}$-terminal region; figure 5) to condensate formation remain to be investigated. The relationship between condensate formation and antiviral activities remains unclear. Nevertheless, it is already clear that subcellular localization of $\mathrm{Mx}$ condensates is an important determinant of antiviral specificity. The key paradigm shift in the last three years has been to move the $\mathrm{Mx}$ field away from the widely prevalent interpretation that MxA associates with intracellular membranes (e.g. the endoplasmic reticulum) towards consideration of $\mathrm{Mx}$ structures as membraneless organelles (MLOs).

\section{Acknowledgements}

This work was supported by a grant from the New York Medical College, and by personal funds of PBS.

\section{References}

Accola MA, Huang B, Masri AA and McNevin MA 2002 The antiviral dynamin family member, MxA, tubulates lipids and localizes to the smooth endoplasmic reticulum. J. Biol. Chem. 277 21829-21835

Alberti S 2017 The wisdom of crowds: regulating cell function through condensed states of living matter. $J$ Cell Sci. 130 2789-2796

Alberti S, Gladfelter A and Mittag T 2019 Considerations and challenges in studying liquid-liquid phase separation and biomolecular condensates. Cell 176 419-434

Banani SF, Lee HO, Hyman AA and Rosen MK 2017 Biomolecular condensates: organizers of cellular biochemistry. Nat. Rev. Mol. Cell Biol. 18 285-298

Boeynaems S, Holehouse AS, Weinhardt V, Kovacs D, Lindt JV, et al. 2019 Spontaneous driving forces give rise to protein-RNA condensates with coexisting phases and complex material properties. Proc. Natl. Acad. Sci. USA $1167889-7898$

Boke E, Ruer M, Wuhr M, Coughlin M, Lemaitre R, et al. 2016 Amyloid-like self-assembly of a cellular compartment. Cell 166 637-650

Busnadiego I, Kane M, Rihn S, Preugchau HF, Hughes J, et al. 2014 Host and viral determinants of Mx2 antiretroviral activity. J. Virol. 88 7738-7752

Davis D, Yuan H, Yang YM, Liang FX and Sehgal PB 2018 Interferon-alpha- induced cytoplasmic MxA structures in hepatoma Huh7 and primary endothelial cells. Contemp Oncol (pozn) 22 86-94

Davis D, Yuan H, Liang FX, Yang YM, Westley J, et al. 2019 Human antiviral protein MxA forms novel metastable membraneless cytoplasmic condensates exhibiting rapid reversible tonicity- driven phase transitions. J. Virol. 93 e01014-e01019

Delarue M, Brittingham GP, Pfeffer S, Surovtsev IV, Pinglay $\mathrm{S}$, et al. 2018 mTORC1 Controls phase separation and the biophysical properties of the cytoplasm by tuning crowding. Cell 174 338-349

Desai SM 1970 Sarcoma blockade in vivo: rabies-Rous system in chickens. Nature 228 460-461

Desai SM, Sehgal PB, Nanavati AND and Shirodkar MV 1973 A rabies-induced serum factor inhibiting Rous sarcoma virus in chickens. J. Gen. Virol. 19 285-293

Dick A, Graf L, Olal D, von der Malsburg A, Gao S, et al. 2015 Role of nucleotide binding and GTPase domain dimerization in dynamin-like myxovirus resistance protein A for GTPase activation and antiviral activity. J. Biol. Chem. 290 12779-12792

Ditlev JA, Case LB and Rosen MK 2018 Who's in and who's out - compositional control of biomolecular condensates. J. Mol. Biol. 430 4666-4684

Du M and Chen ZJ 2018 DNA-induced liquid phase condensation of cGAS activates innate immune signaling. Science 361 704-709 
Engelhardt OG, Sirma H, Pandolfi PP and Haller O 2004 Mx1 GTPase accumulates in distinct nuclear domains and inhibits influenza A virus in cells that lack promyelocytic leukemia protein nuclear bodies. J. Gen. Virol. 85 2315-2326

Gomes E and Shorter J 2019 The molecular language of membraneless organelles. J. Biol. Chem. 294 7115-7127

Goujon C, Moncorge O, Bauby H, Doyle T, Ward CC, et al. 2013 Human Mx2 is an interferon-induced post-entry inhibitor of HIV-1 infection. Nature $\mathbf{5 0 2} 559-562$

Haller O and Kochs G 2002 Interferon-induced Mx proteins: dynamin-like GTPases with antiviral activity. Traffic 3 710-717

Haller O, Arnheiter H, Gresser I and Lindenmann J 1979 Genetically determined, interferon-dependent resistance to influenza virus in mice. J. Exp. Med. 149 601-612

Haller O, Staeheli P and Kochs G 2007 Interferon-induced $\mathrm{Mx}$ proteins in antiviral host defense. Biochimie $\mathbf{8 9}$ 812-818

Haller O, Staeheli P, Schwemmle M and Kochs G 2015 Mx GTPases: dynamin- like antiviral machines of innate immunity. Trends Microbiol. 23 154-163

Haller O, Arnheiter H, Pavloviv J and Staehli P 2018 The discovery of the antiviral resistance gene Mx: a story of great ideas, great failures, and some success. Annu Rev. Virol. $533-51$

Henle W 1950 Interference phenomena between animal viruses: a review. J. Immunol. 64 203-236

Ho M 1962 Interferons. New Engl. J. Med. 266 13131318

Isaacs A and Lindenmann J 1957 Virus interference. I. The interferon. Proc. R. Soc. Lond. B 147 258-267

Isaacs A, Lindenmann J and Valentine RC 1957 Virus interference. II. Some properties of interferon. Proc. R. Soc. Lond. B 147 268-273

King MC, Raposo G and Lemmon MA 2004 Inhibition of nuclear import and cell-cycle progression by mutated forms of the dynamin-like GTPase MxB. Proc. Natl. Acad. Sci. USA 101 8957-8962

Kochs G, Janzen C, Hohenberg H and Haller O 2002 Antivirally active MxA protein sequesters La Crosse virus nucleocapsid protein into perinuclear complexes. Proc. Natl. Acad. Sci. USA 99 3153-3158

Lindenmann J 1962 Resistance of mice to mouse-adapted influenza A virus. Virology 16 203-204

Lindenmann J 1964 Inheritance of resistance to influenza virus in mice. Proc. Soc. Exp. Biol. Med. 116 506-509

Lindenmann J, Lane CA and Hobson D 1963 The resistance of A2G mice to myxoviruses. J. Immunol. $90942-$ 951

Ma W and Mayr C 2018 A membraneless organelle associated with the endoplasmic reticulum enables 3'
UTR-mediated protein-protein interactions. Cell $\mathbf{1 7 5}$ 1492-1506

Milovanovic D, Wu Y, Bian X and De Camilli P 2018 A liquid phase of synapsin and lipid vesicles. Science $\mathbf{3 6 1}$ 604-607

Mitrea DM and Kriwacki RW 2016 Phase separation in biology, functional organization of a higher order. Cell Commun. Signal

Nigg PE and Pavlovic J 2015 Oligomerization and GTPbinding Requirements of MxA for viral target recognition and antiviral activity against Influenza A Virus. J. Biol. Chem. 290 29893-29906

Rai AK, Chen JX, Selbach M and Pelkmans L 2018 Kinasecontrolled phase transition of membraneless organelles in mitosis. Nature 559 211-216

Sabari BR, Dall'Agnese A, Boija A, Klein IA, Coffey EL, et al. 2018 Coactivator condensation at super-enhancers links phase separation and gene control. Science

Sadler AJ and Williams B 2011 Dynamiting viruses with MxA. Immunity 35 491-493

Sehgal PB 2019 Biomolecular condensates in cancer cell biology: interleukin-6- induced cytoplasmic and nuclear STAT3/PY-STAT3 condensates in hepatoma cells. Contemp. Oncol. (pozn) 23 16-22

Sehgal PB, Westley J, Lerea KM, DiSenso-Browne S and Etlinger JD 2020 Biomolecular condensates in cell biology and virology: phase-separated membraneless organelles (MLOs). Anal. Biochem. 597113691

Sehgal PB, Yuan H, Scott MF, Deng Y, Liang FX, et al. 2020 Murine GFP-Mx1 forms phase-separated nuclear condensates and associates with cytoplasmic intermediate filaments: novel antiviral activity against vesicular stomatitis virus. J. Biol. Chem. 597113691

Shiina N 2019 Liquid- and solid-like RNA granules form through specific scaffold proteins and combine into biphasic granules. J. Biol Chem. $294 \quad 3532-$ 3548

Shin Y and Brangwynne CP 2017 Liquid phase condensation in cell physiology and disease. Science 357 eaaf 4382

Shirodkar MV 1965 The blocking effect of West Nile virus on production of sarcoma by Rous virus in chickens. $J$. Immunol. 95 1121-1128

Steiner F and Pavlovic J 2020 Subcellular localization of $\mathrm{MxB}$ determines its antiviral potential against influenza virus. J. Virol. 94 e00125-e220

Stertz S, Reichelt M, Krunse-Locker J, Mackenzie J, Simpson JC, Haller O and Kochs G 2006 Interferoninduced, antiviral human MxA protein localizes to a distinct subcompartent of the smooth endoplasmic reticulum. J. Interferon Cyt. Res. $26 \quad 650-$ 660

Verhelst J, Hulpiau P and Saelens X 2013 Mx proteins: antiviral gatekeepers that restrain the uninvited. Microbiol. Mol. Biol. Rev. 77 551-566 
Yuan H and Sehgal PB 2016 MxA Is a novel regulator of endosome-associated transcriptional signaling by bone morphogenetic proteins 4 and 9 (BMP4 and BMP9). PLoS One 11 e0166382

Zürcher T, Pavlovic J and Staeheli P 1992a Nuclear localization of mouse Mx1 is necessary for inhibition of influenza virus. J. Virol. 66 5059-5066

Corresponding editor: Kundan Sengupta
Zürcher T, Pavlovic J and Staeheli P 1992b Mechanism of human MxA protein action: variants with changed antiviral properties. $E M B O \quad J . \quad 11 \quad 1657-$ 1661

Zürcher T, Pavlovic J and Staeheli P 1992c Mouse Mx2 protein inhibits vesicular stomatitis virus but not influenza virus. Virology 187 796-800 